\title{
Comparison of unipolar and bipolar ventricular paced evoked responses
}

\author{
M W Baig, J C Cowan, E J Perrins
}

\begin{abstract}
Objectives-To study the differences between endocardial bipolar and unipolar ventricular paced evoked responses and surface electrocardiograms.

Patients-10 patients with conduction system disease awaiting insertion of a permanent pacemaker were studied with temporary ventricular pacing from the right ventricular apex.

Main outcome measure-Comparison of the durations of the QRS complexes and QTa and QTe intervals of the endocardial bipolar paced evoked response and the surface electrocardiogram with those of the reference unipolar paced evoked response.

Results-By comparison with the unipolar reference, the mean durations of the QRS complexes of the bipolar signal and the surface electrocardiogram were $41 \cdot 8 \%$ and $132 \cdot 1 \%$ respectively. The mean QTa interval was $85.9 \%$ and $112.2 \%$ respectively and the mean QTe interval was $86.9 \%$ and $109 \cdot 5 \%$ respectively. All these differences were significant. The amplitudes of the unipolar QRS complexes and $T$ waves were significantly larger than those recorded in the bipolar configuration.

Conclusions-Differences between the unipolar and bipolar ventricular paced evoked responses are significant. The time course of the unipolar signal is closer to that of the surface electrocardiogram. This indicates that the unipolar paced evoked response does not reflect local electrophysiological events, as has been suggested previously.
\end{abstract}

(Br Heart J 1992;68:398-402)

The ventricular paced evoked response was first described in 1981 and has been used to provide the sensor for the QT sensing, rate adaptive pacemaker.' The paced evoked response is recorded conventionally as a unipolar signal with the negative pole within the heart and the positive pole, which is usually the case of the pacemaker, at a variable distance on the body's surface. Previous studies have noted that the interval between the pacing stimulus and the apex of the evoked $\mathrm{T}$ wave corresponds roughly to the time to $90 \%$ repolarisation of a locally recorded monophasic action potential. ${ }^{23}$

Consequently, it has been assumed that the paced evoked response reflects the duration of local action potential and that it might provide an easy measurement of local ventricular repolarisation as an alternative to the monophasic action potential. By its nature, however, a unipolar electrogram contains both local and far field components. ${ }^{4}$ Previous investigators have cautioned against using unipolar signals to determine local electrophysiological events. ${ }^{5}$ By contrast, both poles of a bipolar system are located within the same cardiac chamber, and this type of electrogram is inherently more suited to the assessment of local depolarisation and repolarisation.

The aims of this study were to compare the characteristics of simultaneously recorded bipolar and unipolar paced evoked responses and to assess the relative contributions of local and global myocardial depolarisation and repolarisation to the morphology of the unipolar paced evoked response.

\section{Patients and methods}

PATIENT SELECTION

Ten patients (mean age 66.3 years; six men) were studied. Five had complete heart block, and the rest had sick sinus syndrome. In all cases standard ( $6 \mathrm{~F}$ USCI) bipolar temporary pacing electrodes had been inserted before permanent pacemakers were implanted, and temporary pacing was performed from the right ventricular apex. None of the patients had a history of myocardial infarction or were taking any drugs likely to affect cardiac electrophysiology. Studies were performed in the postabsorptive state after informed consent had been obtained.

RECORDING OF THE PACED EVOKED RESPONSE A large adhesive defibrillator pad (surface area $178 \mathrm{~cm}^{2}$ ) placed over the right scapula served as the remote, indifferent electrode. Unipolar stimulation was used in all cases. This is the standard method of cardiac stimulation as the wide distance between electrodes reduces residual polarisation potentials and facilitates sensing of the paced evoked response. A dedicated external pulse generator is needed to record the paced evoked response." This delivers a modified pulse waveform that, together with inbuilt circuitry for automatic postpulse compensation, eliminates the polarisation potential that is generated at the interface between the myocardium and the electrode. ${ }^{78}$ The pulse generator contained two identical sensing amplifiers, which enabled 
simultaneous recording of the unipolar and the bipolar paced evoked responses being recorded between the tip and ring electrodes (separation $10 \mathrm{~mm}$ ) of the pacing lead. The output of the pulse generator was set to twice the diastolic threshold with a pulse duration of $0.5 \mathrm{~ms}$, and the rate was fixed to ensure continuous capture.

For patients with complete heart block the pacing rate was 70 beats/min. For patients who had sinoatrial disease faster rates were necessary to ensure continuous pacing (80-110 beats $/ \mathrm{min}$ ), although the minimum overdrive pacing rate was used to minimise the potential adverse haemodynamic effects and their associated autonomic reflexes. These may occur with ventricular pacing in this group of patients and could have affected the values studied. Electrograms were recorded on a chart recorder (Gould ES 1000) at a paper speed of $100 \mathrm{~mm} / \mathrm{s}$ after a minimum of three minutes of pacing to ensure stabilisation of the myocardial repolarisation time. ${ }^{910}$

The endocardial electrograms were compared with the complexes of the standard 12 lead surface electrocardiogram recorded in turn together with the simultaneous unipolar and bipolar paced evoked responses. There is no generally accepted method for selecting leads for measuring the QT interval. Therefore, to standardise the measurement technique in our study the criteria for selecting the surface electrocardiogram lead that was to be compared with the paced evoked responses were that it should show a clearly defined $\mathrm{T}$ wave and the least difference in total duration when compared with the unipolar paced evoked response. The second criterion used minimised the probability of detecting a significant difference between the QT intervals of the evoked response and the surface electrocardiogram. Consequently, measurements of the surface electrocardiogram were made most frequently from leads V1 and V2. Right ventricular apical pacing produced the expected pattern of left axis deviation and complete left bundle branch block in all cases. ${ }^{11}$

\section{DEFINITIONS AND MEASUREMENTS \\ Evoked QRS complex}

In the paced evoked response the initial deflection after the pacing stimulus is negative (evoked QRS complex) and denotes spread of the depolarisation wavefront away from the stimulating electrode; it is followed by a positive evoked $\mathrm{T}$ wave (fig 1). The duration of the evoked QRS is a measure of total ventricular depolarisation time in the area of the heart subtended by a sensing dipole, and it depends on the separation of the dipole. ${ }^{12}$ The duration of the evoked QRS, defined as the interval between the pacing stimulus and the end of the $S$ wave-that is, the point when the isoelectric line is crossed ${ }^{13}$ was measured for the simultaneous bipolar and unipolar paced evoked responses and from the surface electrocardiogram lead.

Evoked QT intervals

The QT interval is the standard clinical measure of ventricular repolarisation time. It

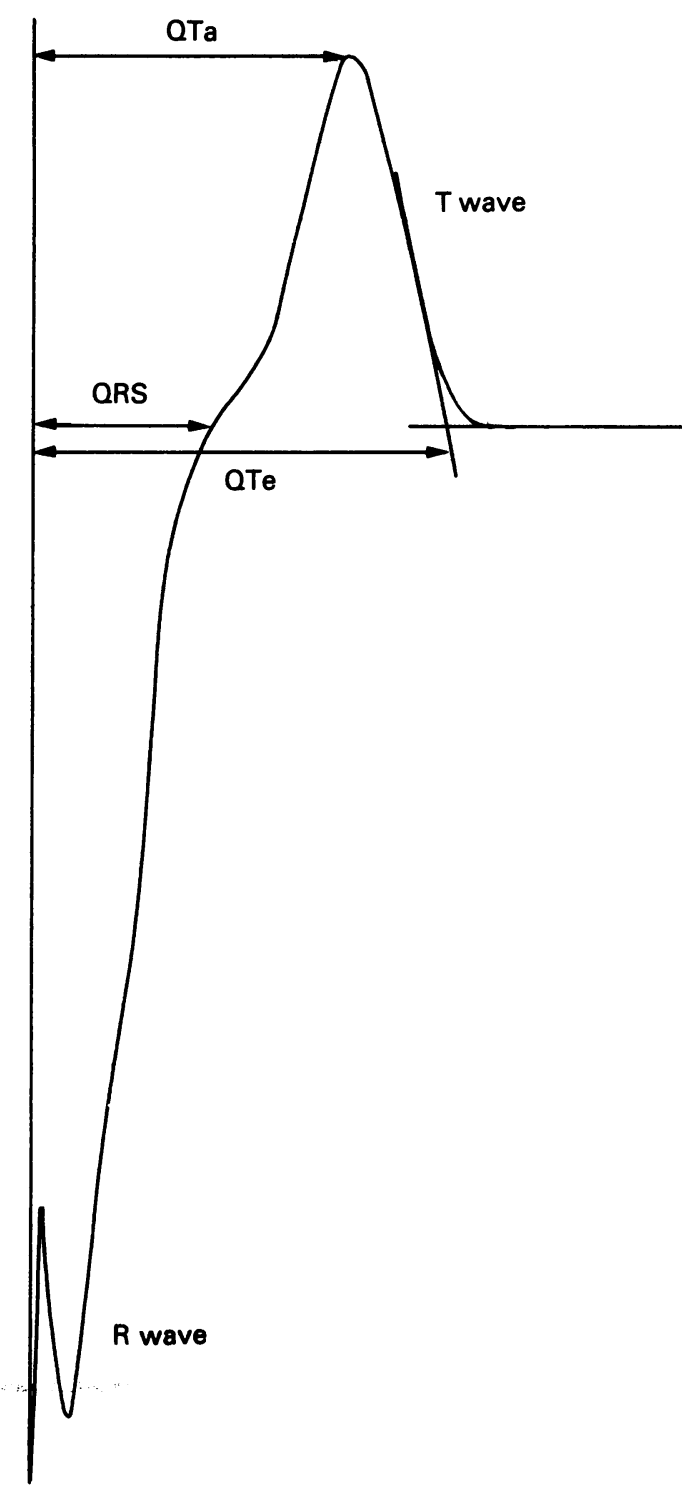

Figure 1 Components of unipolar ventricular paced evoked response, showing definitions of $Q R S, Q$ to apex of $T(Q T a)$, and $Q$ to end of $T(Q T e)$ intervals.

was measured from the pacing artefact to the terminal $T$ wave deflection (QTe) by standard criteria, in which the end of the $T$ wave is defined as the intersection of the tangent drawn through the point of maximum downslope of the $T$ wave with the isoelectric line. ${ }^{14} 15$ The QTa was defined as the interval between the pacing stimulus and the apex of the $T$ wave (fig 1). ${ }^{15}$

\section{Amplitude of evoked QRS complexes and T waves}

The amplitudes of the evoked QRS complexes and $T$ waves were measured from the isoelectric line for the simultaneous unipolar and bipolar signals and the average value calculated by reference to a $5 \mathrm{mV}$ calibration signal.

\section{STATISTICAL ANALYSIS}

The -simultaneous evoked QRS and QT intervals of the bipolar and unipolar paced evoked responses and the selected lead of the surface electrocardiogram for five consecutive beats were measured and the average interval calculated. The QRS and QT intervals of the 
Table 1 Standardised durations of $Q R S$ complexes and $Q T$ intervals of simultaneously recorded bipolar paced evoked response (PER) and surface electrocardiogram (ECG) with respect to unipolar PER (values are percentages of PER)

\begin{tabular}{|c|c|c|c|c|c|}
\hline & \multicolumn{2}{|c|}{ Bipolar } & \multirow[b]{2}{*}{ Unipolar } & \multicolumn{2}{|c|}{ Surface ECG } \\
\hline & Mean & ( $S D$, range) & & Mean & $(S D$, range $)$ \\
\hline $\begin{array}{l}\text { QRS } \\
\text { QTa } \\
\text { QTe }\end{array}$ & $\begin{array}{l}41 \cdot 8 \\
85.9 \\
86.9\end{array}$ & $\begin{array}{r}(16.4,22-69) \\
(1.9,83-88) \\
(3 \cdot 6,83-93)\end{array}$ & $\begin{array}{l}100 \\
100 \\
100\end{array}$ & $\begin{array}{l}132 \cdot 1 \\
112 \cdot 2 \\
109 \cdot 5\end{array}$ & $\begin{array}{r}(16 \cdot 3,101-158) \\
(3 \cdot 1,109-115) \\
(5 \cdot 8,102-118)\end{array}$ \\
\hline
\end{tabular}

Analysis of variance with $p<0.01$ for comparison of $Q R S$ intervals gave $F_{\text {crit }}=5.49$ and $F_{\text {obs }}=117.6$ and for comparison of $Q T$ intervals, $F_{\text {crit }}=5.49$ and for $Q T a F_{o b s}=161.8$ and for QTe $F_{\text {obs }}=83.4$ (see text for explanation of terms).

Table 2 Comparisons of amplitudes ( $m V$ ) of $Q R S$ complexes and $T$ waves of simultaneously recorded bipolar and unipolar paced evoked response (PERs) by paired $\mathrm{t}$ test

\begin{tabular}{lllllll}
\hline & \multicolumn{2}{l}{ Bipolar PER } & & \multicolumn{2}{l}{ Unipolar PER } & \\
\cline { 2 - 3 } & Mean & $(S D$, range $)$ & & Mean & $(S D$, range $)$ & p Value \\
\hline QRS complex & 9.9 & $(3 \cdot 8,3 \cdot 7-17.6)$ & & 13.5 & $(3.4,8.3-19.8)$ & $<0.001$ \\
Twave & 2.6 & $(1.4,1.1-4.8)$ & & 5.7 & $(1.9 .2 .6-7 \cdot 1)$ & $<0.002$ \\
\hline
\end{tabular}

bipolar paced evoked response and the surface electrocardiogram were standardised as a percentage of the values of the unipolar paced evoked response to take account of differences in the pacing rate between patients. The difference between intervals was assessed by one way analysis of variance. Results were assessed by comparing the $\mathrm{p}$ values and the computed value of the test $\left(f_{\text {obs }}\right)$ with the critical value $\left(F_{\text {crit }}\right)$. The value of $F_{\text {crit }}$ for these data was 5.49; values of $F_{\text {obs }}$ greater than this indicate a significant difference. Table 1 summarises the mean (SD) values of the intervals under study.

The mean amplitudes of the evoked QRS complexes and $T$ waves of the bipolar and unipolar evoked responses were calculated with reference to the $5 \mathrm{mV}$ test signal. The results were analysed with Student's paired $t$ test and are expressed as means (SD). Significance was inferred if $\mathrm{p}<0.01$, and the $95 \%$ confidence intervals $(95 \% \mathrm{CI})$ were calculated (table 2$)$.

\section{Results}

COMPARISON OF EVOKED QRS DURATIONS

The duration of the measured QRS interval increased progressively from the bipolar to the

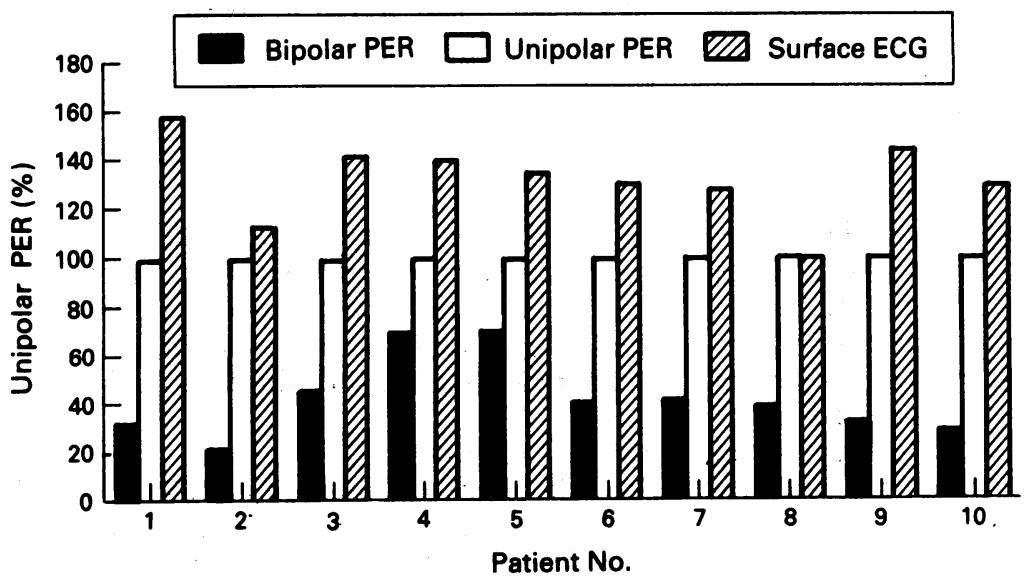

Figure 2 Relative durations of QRS complexes of bipolar and unipolar paced evoked responses (PER) and surface electrocardiogram (ECG). Values have been standardised with reference to unipolar PER. unipolar paced evoked response and from this to the measurements made from the surface electrocardiogram (table 1 and fig 2). Analysis of variance indicated that the durations of the QRS complexes of the bipolar paced evoked response and the surface electrocardiogram differed highly significantly from that of the unipolar evoked response $\left(F_{\text {obs }}=117.6\right.$, p $<0.0001$, where $F_{\text {crit }}=5.49$ at the $5 \%$ level). The differences in absolute terms were less between the unipolar paced evoked response and the surface electrocardiogram $(32 \cdot 1 \%)$ than those between the two paced evoked responses $(58.2 \%)$. This suggests that the unipolar paced evoked response more closely resembles the surface electrocardiogram and therefore reflects a depolarisation wavefront recorded over a larger area than the local electrical activity recorded by the bipolar paced evoked response.

COMPARISON OF THE EVOKED QT INTERVALS

The changes in the QTa and QTe intervals showed a similar pattern to that for the duration of the QRS interval (table 1, fig 3a and b). The differences in the mean durations of the QTa and QTe intervals of the bipolar evoked response (QTa $14 \cdot 1 \%$, QTe $13 \cdot 1 \%$ ) and the surface electrocardiogram (QTa $12 \cdot 2 \%$, QTe $9.5 \%$ ) were similar when compared with the unipolar evoked response. For the QRS component these differences in the QT intervals were highly significantly different (for QTa intervals $F_{o b s}=161.8, p<0.0001$; for QTe intervals $\mathrm{F}_{\text {obs }}=83.4, \mathrm{p}<0.0001$, where $\mathrm{F}_{\text {crit }}=5 \cdot 49$ ).

AMPLITUDES OF THE EVOKED QRS AND T WAVES

The difference between the mean amplitudes of the bipolar and unipolar paced evoked QRS complexes was $-3.49 \mathrm{mV}$, with a $95 \% \mathrm{CI}$ of -4.7 to $-2.3 \mathrm{mV}(\mathrm{p}<0.001)$. Similar significant differences were found for the mean evoked $T$ wave amplitudes. The difference between the mean amplitudes of the bipolar and unipolar evoked $\mathrm{T}$ waves was $-3.08 \mathrm{mV}$, with a $95 \%$ CI of -4.4 to $-1.7 \mathrm{mV}$ $(\mathrm{p}<0.002)$

\section{Discussion}

The main bioelectrical events of ventricular electrograms are the intracardiac QRS complex, the intracardiac $T$ wave, and any possible superimposed injury current. The first two components have been discussed in detail. Six of the temporary electrodes had been in place for more than $\mathbf{4 8}$ hours, and any injury current had probably resolved. ${ }^{16}$ Our previous work in which the reproducibility of the relation between the paced QT interval and rate has been examined with an interval of not less than 24 hours has shown no significant difference in the morphology of the paced evoked response. Furthermore, ST segment shift is unlikely to have affected the measurements made in this study.

Electrocardiograms are the net result of the electric currents generated by ion exchange across cardiac cell membranes. The heart gen- 

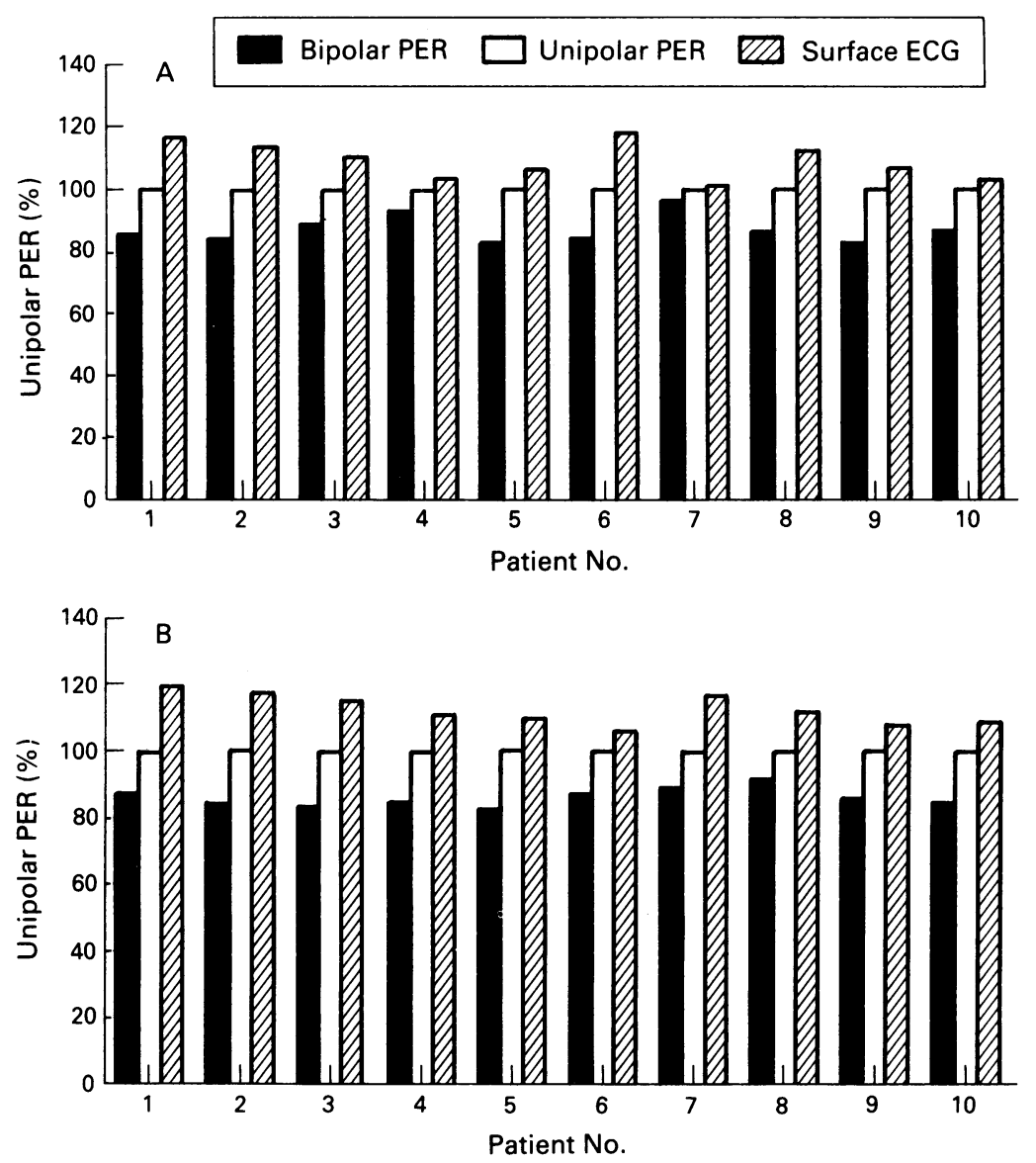

Figure 3 Relative durations of ( $A$ ) QTa and ( $B$ ) QTe intervals of bipolar and unipolar paced evoked responses (PERs) and surface electrocardiogram (ECG). Values have been standardised with reference to unipolar PER.

erators complex signals, and the addition of a surface electrode introduces further complexity; the intervening thorax and boundaries (skin-air, and intracavity blood mass within the heart) are non-homogeneous conductors, and the irregular form of the torso will affect the shape and amplitude of the potentials recorded. ${ }^{17-19}$ Lewis and Rothschild were the first to recognise that unipolar electrograms contained both local components (intrinsic deflection) and signals generated by more remote tissue (extrinsic deflection). ${ }^{4}$

Later studies have shown that the shapes and peak to peak voltages of unipolar electrograms are influenced mainly by the superposition effects of distant excitation waves and not by local events. ${ }^{2021}$ Blanchard et al have shown that the electrical activity associated with activation of the left ventricle has a significant effect on the right ventricular unipolar electrogram even after the two ventricles have been disconnected electrically by a disarticulation procedure. ${ }^{22}$ The differences in the durations of the evoked QRS complexes for the bipolar and unipolar packed evoked responses reported in our study are similar to those found in unpaced electrograms, ${ }^{23}$ and the longer duration of the unipolar signal indicates that it reflects ventricular activation and repolarisation over a wider area.

The preliminary evidence that the unipolar evoked response might reflect a dominance of local electrophysiological changes around the stimulating electrode was based largely on the finding that the interval between the pacing spike and the apex of the evoked $\mathrm{T}$ wave coincided roughly with the time to $90 \%$ repolarisation of a locally recorded monophasic action potential. Furthermore, the application of physiological and pharmacological stimuli known to affect the duration of the monophasic action potential caused a parallel change in the duration of the interval from stimulus to apex of the evoked $T$ wave. As the $T$ wave represents the summation at the body surface of the electrical forces during repolarisation, however, any induced changes in the monophasic action potential might be expected to result in similar changes in the $\mathrm{T}$ wave. ${ }^{2425}$ Indeed, evidence for this has been reported. ${ }^{26}$

The results of this study are consistent with other comparisons of unipolar and bipolar electrograms, although only limited information is avilable concerning the electrical characteristics of the unipolar paced evoked response. ${ }^{27}$ The longer duration of the QRS and QT intervals of the unipolar signal and its closer approximation to the surface electrocardiogram than to the bipolar paced evoked response support the hypothesis that the unipolar evoked response has a significant signal content from more distal portions of the heart, probably including contralateral ventricular electrical activity. ${ }^{28-30} \mathrm{~A}$ bipolar sensing system selectively cancels far field signals and therefore is more likely to represent local depolarisation and repolarisation, and the bipolar ventricular paced evoked response might become more widely used as a measure of local cardiac electrical phenomena.

This is the first study that compares the morphologies and durations of the bipolar and unipolar ventricular paced evoked responses. Our results indicate that, contrary to previously published data, the unipolar paced evoked response is significantly influenced by far field electrical phenomena and is unlikely to give quantitative information about local myocardial depolarisation and repolarisation; these three electrograms should therefore be considered as separate entities. Further studies of the duration of local action potentials that use the paced evoked response are probably best conducted with a bipolar sensing configuration. The larger amplitude of the unipolar paced evoked $\mathrm{T}$ wave compared with the simultaneous bipolar signal, however, implies that its detection in the QT sensing pacemaker is more certain with the first sensing configuration.

1 Rickards AF, Norman J. Relation between QT interval and heart rate. New design of physiologically adaptive cardiac pacemaker. Br Heart J 1981;45:56-61.
Donaldson RM, Rickards AF. Evaluation of drug-induced Donaldson RM, Rickards AF. Evaluation of drug-induced
changes in myocardial repolarisation using the paced evoked response. Br Heart J 1982;48:381-7.

3 Donaldson RM, Taggart P, Nashat F, Abed J, Rickards AF, Noble D. Study of the electrophysiolsogical effects of early or subendocardial ischaemia with intracavitary electrodes in the dog. Clin Sci 1983;65:579-88.

4 Lewis T, Rothschild MA. The excitatory process in the dog's heart. Part II. The ventricles. Phil Trans $R$ Soc Lond 1915;206:181-226.

5 Scher AM, Young AC. Ventricular depolarization and the genesis of the QRS. Ann NY Acad Sci 1957;6:768-78.

6 Donaldson RM, Rickards AF. The ventricular endocardial paced evoked response. PACE 1983;6:253-9. 
7 Mansfield PB. Myocardial stimulation: the electrochemistry of electrode-tissue coupling. Am J Physiol 1967;212: 1475-88.

8 Baig W, Walton C, Boute W, Perrins J. Comparison of two techniques for reduction in post-stimulus electrode polarization [abstract]. PACE 1989;12:677.

9 Boyett MR, Jewell BR. A study of the factors responsible for rate-dependent shortening of the action potential in rate-dependent shortening of the action potential in
mammalian ventricular muscle. $J$ Physiol (Lond) 1978; 285:359-80.

10 Arnold L, Page J, Attwell D, Cannell M, Eisner DA. The dependence on heart rate of the human ventricular action potential duration Cardiovasc Res 1982;16:547-51.

11 Castellanos A, Ortiz JM, Pastis N, Castillo C. The electrocardiogram in patients with pacemakers. Prog Cardiovasc Dis 1970;13:190-209.

12 Geselowitz DG. Dipole theory in electrocardiography. Am J Cardiol 1964;14:301-6.

13 DeCaprio V, Hurzeler P, Furman S. A comparison of unipolar and bipolar electrograms for cardiac pacemaker sensing. Circulation 1977;56:750-5.

14 Lepeschkin E, Surawicz B. The measurement of the Q-T interval of the electrocardiogram. Circulation 1952;6: interval

15 Simonson E, Cady LD, Woodbury $M$. The normal QT interval. Am Heart J 1962;63:743.

16 Gordon AG, Vagueiro MC, Barrold SS. Endocardial electrograms from pacemaker catheters. Circulation electrograms

17 Holland RP, Arnsdorf MF. Solid angle theory and the electrocardiogram: physiologic and quantitative interpretations. Prog Cardiovasc Dis 1977;19:431-57.

18 Corbin V, Scher AM. The canine heart as an electrocardiographic generator. Dependence on cardiac cell orientation. Circ Res 1977;41:58-67.

19 Selvester RH, Solomon JC, Gillespie TL. Digital computer model of a total body electrocardiographic surface map.
Circulation 1968;38:684-90.

20 Spach MS, Barr RC. Ventricular intramural and epicardial potential distributions during ventricular activation and repolarization in the intact dog. Circ Res 1975;37:243-57.

21 Steinhaus BM. Estimating cardiac transmembrane activation and recovery times from unipolar and bipolar extracellular electrograms: a simulation study. Circ Res 1989;64:449-62.

22 Blanchard SM, Damiano R, Asano T, Smith W, Ideker RE Lowe JE. The effects of distant cardiac electrical events on Lowe JE. The effects of distant cardiac electrical events on Trans Biomed Eng 1987;7:539-46.

23 Furman S, Hurzeler P, De Caprio V. Cardiac pacing and pacemakers. III. Sensing the cardiac electrogram. $A m$ Heart $J$ 1977;93:794-801.

24 Van Dam RT, Durrer D. The $T$ wave and 'ventricular repolarization. Am J Cardiol 1964;14:294-300.

25 Harumi K, Burgess MJ, Abildskov. A theoretic model of the T wave. Circulation 1966;34:657-68.

26 Autenrith G, Surawicz B, Kuo CS, Arita M. Primary T wave abnormalities caused by uniform and regional shortening of the ventricular monophasic action potential in the dog. Circulation 1975:51:668-76.

27 Boute W, Wittkampf FHM. Morphology of evoked intracardiac potentials: their importance in IPGs [abstract] PACE 1988;11:805-10.

28 Dower GE, Geddes MA. True transmembrane potential curve from the surface of the guinea pig ventricle: its curve from the surface of the guinea pig ventricle: its relation

29 Adams E. The electrode-biointerface: sensing. In: Barold S, Mugica J, eds' New perspectives in cardiac pacing. Moun Kisco, New York: Futura, 1988;17-25.

30 Blanchard SM, Damiano RJ, Smith WM, Ideker RE, Low $\mathrm{JE}$. Interpolating unipolar epicardial potentials from electrodes separated by increasing distances. $P A C E$ 1989;12:1938-55. 